Historic, Archive Document

Do not assume content reflects current scientific knowledge, policies, or practices. 


\section{TO OUR CUSTOMI}

We warrant all our plants to be rue and in good growing condition at time of shipping, but are not responsible for any damage or loss that may occur in transit.

Seeds are prepaid by us. Plants by express must be paid by purchaser. If plants are ordered by freight add enough to cost of plants to prepay charges.

Packing, as is customary, we are making a charge for this service only to cover cost of material used.

Should any mistake occur (which we do our best to avoid) we will either duplicate your order or refund your money, should the fault lie with us.

All complaints must be made within five days on receipt of goods, or we cannot consider the same. But in no case can we be responsible for the crop.

Orders from unknown parties must be accompanied by cash or reference. All bills from parties of approved credit due the first of the following month. In all cases where the bilis are not paid when due, we reserve the privilege of drawing on same without further notice.

Order as early as convenient, stating date of shipment, and we will reserve stock for you and forward as desired. Should you visit Santa Rosa during the growing season we would be pleased to have you call and see our stock.

Thanking our many customers for their kind patronage and hoping for a continuance of same, we are

Respectfully,

R. F. D. No. 4

FRED GROHE CO.

Santa Rosa, Cal. 


\section{PLANTS}

ACHILLEA, The Pearl,

Doz. $\quad 100$

From field .................. $.35 \$ 2.50$

ANCHUSA, Dropemore Variety

3 in pots .................... 40.00

ASPARAGUS, Plumosus,

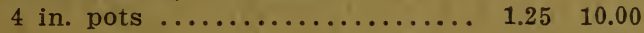

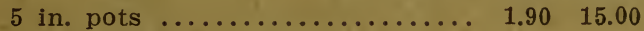

ASPARAGUS, Sprengeri,

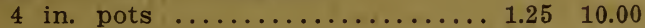

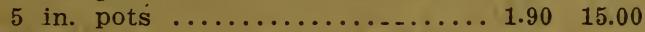

ASTERS, ready in March,

Vick's Branching, all colors:

Strong, transplanted $\ldots \ldots \ldots \ldots \ldots . \quad 1.00$

Per 1000 , assorted, $\$ 8.00$.

BEGONIA, Flowering, Otto Hecker, Agenta Gutta, Diadem, Coral, Metalica.

2 in. pots $\ldots \ldots \ldots \ldots \ldots \ldots \ldots \ldots \ldots . .35-2.50$

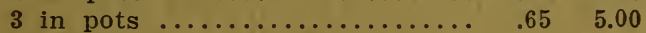

Triumph de Loraine or Double

Vernon,

$21 / 4$ in. pots $\ldots \ldots \ldots \ldots \ldots \ldots \ldots . .40 \quad 2.50$

CYCLAMEN, from English seed,

Five named varieties for Easter

Blooming:

4 in. pots $\ldots \ldots \ldots \ldots \ldots \ldots \ldots \ldots \ldots 2.50 \quad 20.00$

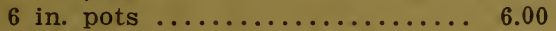

CAMPANULA,

Carpatica, good for edgings or

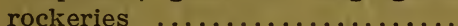

PERSICIFOLIA, Blue or White,

3 in. pots $\ldots \ldots \ldots \ldots \ldots \ldots \ldots \ldots \ldots \ldots \ldots \ldots \ldots+.65 \quad 5.00$

CHRYSANTHEMUMS:

\section{White Varieties}

Mrs. Robinson, Early.

Smith's Advance, Early.

Oconto, Early.

October Frost, Early.

Smith Ideal, Mid-season.

Nellie Pocket, Mid-season.

Timothy Eaton, Mid-season.

White Bonafon, Mid-season.

Moneymaker, Mid-season.

Silver Wedding, Mid- season.

White Good Gracious, Mid-season

White Cloud, Mid-season.

Queen, Mid-season.

Wm. Turner, Mid-season.

Jeannie Nomin, Late.

Chas. Razer, Late. 
Pink Gem, Early.

Unaka, Early.

Pink Ivory, Early.

William Duckham, Mid-season.

Sappho, Mid-season.

Pink Chieftain, Mid-season.

Smith's Sensation, Late.

Helen Frick, Late.

Wells' Late Pink, Late.

Elberon, Late

Crocus, Early

\section{Yellow Varieties}

Roman Gold, Mid-season.

Mrs. Wm. Duckham, Mid-season.

Major Bonaffon, Mid-season.

Golden Eagle, Mid-season.

Dolly Dimple, Late.

Col. Appleton, Late.

\section{Red Shades}

Black Hawk, Mid-season.

Lord Hopetoun, Mid-season.

S. A. Naceur Bey, Mid-season.

A. S. Wright, Late.

Per 1000 , assorted, $\$ 18.00$.

Rooted Cuttings .................30

POMPON CHRYSANTHEMUMS:

White

Baby Margaret.

Jetsu-Jetui, Petals Quilled.

La Purite.

Vita.

\section{Yellow Shades}

Emo, Bronze button variety.

Mrs. Frank Bew, Bronze.

Triumph D'Or, Golden Yellow.

\section{Pink Shades}

Minta, Light Solft Pink.

Allene, Rose Pink.

Western Beauty, Deep Pink.

Red Shades

Julia Lagravere, Dark Rich Red.

Little Bob, Reddish Brown.

Rooted Cuttings $\ldots \ldots \ldots \ldots \ldots \ldots, \quad .30$

CLEMATIS MONTANA, White, 2year-old. Each, $20 \mathrm{c} . \ldots \ldots \ldots \ldots 2.20$

DAISY, Mammoth English (Bellis)

Pink or White $-. . \ldots \ldots \ldots \ldots \ldots, .35$ 
Doz. 100

DELPHINIUM, Gold Medal Hybrids

Runs through all shades of blue from very light to purple:

3 in. pots, will bloom next sea-

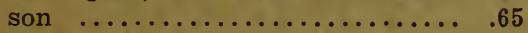

DIGITALIS GLOXINIAEFLORA

Small plants from flats........... . $30 \quad 2.50$

\section{DRACEANA INDIVISA}

5 or 6 in. pots, 18 inches to 2 feet high, $30 \mathrm{c}$ each.

FEVERFEW, Little Gem, blooms all season, $2 \frac{1}{4}$ in. pots..................................... 25

3 in. pots $\ldots \ldots \ldots \ldots \ldots \ldots \ldots \ldots \ldots . .50 \quad 4.00$

FUSCHIA, in assortment, $21 / 4$ in. pots ........................

GAILLARDIA, Kelway's Hybrids $21 / 4$ in. pots $\ldots \ldots \ldots \ldots \ldots \ldots \ldots \ldots \ldots .35 \quad 2.50$

3 in. pots $\ldots \ldots \ldots \ldots \ldots \ldots \ldots \ldots \ldots .6 \%$.60 4.50

GEUM

Mrs. Bradshaw .............. .30 2.50

\section{GLADIOLUS}

Large Bulbs; assorted......... .30 2.00

\section{HELIOTROPE,}

Dark, Czar, Czarina, Madame Braunt;

Light, Giant White.

$21 / 4$ in. pots $\ldots \ldots \ldots \ldots \ldots \ldots \ldots \ldots .25$

3 in. pots $\ldots \ldots \ldots \ldots \ldots \ldots \ldots \ldots . .50 \quad 5.00$

HOLLYHOCKS, Charter's Prize .Strain, the best double in white. pink and red, yellow and maroon, year old plants $\ldots \ldots \ldots \ldots \ldots \ldots \ldots 1.00$

$21 / 4$ in. pots $\ldots \ldots \ldots \ldots \ldots \ldots \ldots . .30 \quad 2.50$

HYDRANGEA OTASKA,

16 to 20 crowns, $2 \frac{1}{2}$ to 3 feet

high, $75 \mathrm{c}$ each $\ldots \ldots \ldots \ldots \ldots \ldots .9 .00$

2 to 3 crowns, $20 \mathrm{c}$ each.......... 2.00

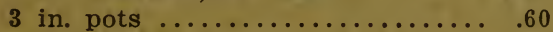

Rubra or Black Stem

16 to 20 crowns, $2 \frac{1}{2}$ to 3 feet high, $65 \mathrm{c}$ each ................... 7.50

LOBELIA, single or double blue, $21 / 4$ in. pots $\ldots \ldots \ldots \ldots \ldots \ldots \ldots .25$

MARGUERITE, white and yellow $2 \frac{1}{4}$ in. pots $\ldots \ldots \ldots \ldots \ldots \ldots \ldots \ldots .35 \quad 2.50$

Mrs. Saunders, new double........ $.35 \quad 2.50$ 
PENSTEMON, Hybridum Grandiflórum, a very choice large flowering strain, flowers often 2 inches across, runs through many colors, such as red, pink, white, maroon, white edged with pink or lavender, and many other variations.

All Colors Mixed:

2 in. pots, ready in March........

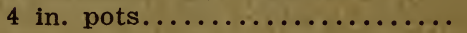

Named varieties in separate colors.

Sonoma, Bright Red, pure white throat.

Santa Rosa, Bright Red, throat splashed and penciled carmine.

Fulton, Pure White throat, edged lavender.

California, Pure White throat, edged pink.

Windsor, Bright Pink, throat splashed and striped red.

Ukiah, Maroon, striped throat, $2 \frac{1}{4}$ in. pots, ready in March......

4 in. pots of Sonoma, Red, Fulton, White Lavender edge, Windsor, bright Pink, ready now......... 1.00

\section{PETUNIAS:}

Gianta of California, Grohe's Champion Strain. Seedlings from flats.$\ldots \ldots \ldots \ldots \ldots \ldots \ldots \ldots$

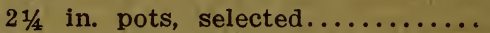

3 in. pots, selected........... .60

Ruffled Giant, Grohe's Champion Strain, seedlings from flats.......

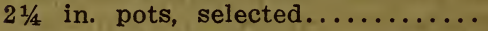

3 in. pots, selected, in bud and bloom in March ............. .65

\section{Frilled and Ruffled Hybrida} Grandiflora Fimbriata. Another Champion Strain:

Seedlings from flats $\ldots \ldots \ldots \ldots, .25$

$21 / 4$ in. pots, selected........... .35

3 in. pots, selected, in bud and bloom in March ................

DOUBLE PETUNIAS, Large Flowering, Fancy Varieties: 
PHLOX, Perennial varieties in good

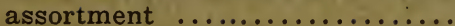

Antonin Mercie, Lavender, white eye.

Benzanson, Red darker eye.

Bridesmaid, White, red eye.

Fraulein von Lassburg, Pure white

Gen. von Hentz, Salmon red, white center.

Von Goethe, Pink, white eye.

Paul Dutry, White splashed pink.

Rubus, Dark rich red.

PHLOX SUBULATA:

Splendid for edging beds or covering graves.

Subulata Purpurea, Lavender.

Subulata Rosea, Pink.

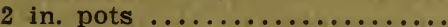

PRIMULA OBCONICA, Gigantea,

in separate colors or mixed.

3 in. pots $\ldots \ldots \ldots \ldots \ldots \ldots \ldots \ldots$

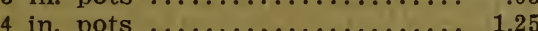

5.00

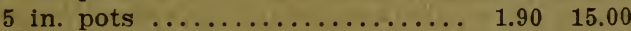

POPPY OR!ENTAL, Large flower-

ing crimson. 3 in. pots............ $.60 \quad 5.00$

PYRETHRUM, Hybridum,

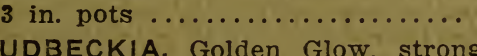
field division .................

SALVIA, Splendens

Seedlings from flats........... .30

$21 / 4$ in. pots, in March.......... .40

SHASTA DAISY, Alaska, California and Westralia, originator's stock. Can be shipped any distance by my improved way of packing. Extra strong field divisions, all of one kind or assorted.............. Per $1000, \$ 32.50$.

SNAPDRAGON, Pink, white, red and yellow, from flats.......... .20

2 in pots, in March . . . ............

STOKESIA CYANEA, Strong field divisions, blue or white......... 50 .50 4.00

\section{STOCKS,}

White, pink, crimson, purple, and lavender ....................... 
VERBENA, Large flowering from rooted cuttings; white, pink, red, purple or assorted, $2 \frac{1 / 4}{4}$ in. pots.... .25

VIOLETS, California Giants

Swanley White

Marie Louise

Per 1000, $\$ 8.00 \ldots \ldots \ldots \ldots \ldots \ldots \ldots$

1.00

\section{SEEDS}

CAMPANULA, Calicanthema, Cup and Saucer. Mixed............

CAMPANULA, Calicanthema, Pink, White or Blue $\ldots \ldots \ldots \ldots \ldots \ldots, \quad .20$

CAMPANULA, Persicifolia ....... .25

DELPHINIUM, Belladonna...... .25

DELPHINIUM,

Gold Medal Hybrids......... . 20

GAILLARDIA, Kelway's Hybrids..

GEUM, Mrs. Bradshaw ..........

HOLLYHOCK, Charter's Prize Strain, Pink, Red, Yellow or

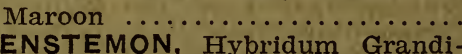
florum, New, large flowering.... PETUNIA, Hybridum, Grandiflorum. Grohe's Champion Strain. Giants of California, $1 / 8$ oz., 3.00 ; $1 / 4$ oz. $\$ 5.00 \ldots \ldots$. 1000 seeds, 50 cents.

Ruffled Giants, $1 / 8 \quad$ oz., $\$ 3.50$, $1 / 4$ oz., $\$ 5.50 \ldots \ldots \ldots \ldots \ldots \ldots \ldots$ 1000 seeds, 75 cents.

Ruffled and Fringed Giants, $1 / 8$ oz., $\$ 3.50 ; 1 / 4$ oz., $\$ 5.50 \ldots \ldots \ldots$. 1000 seeds 75 cents.

POPPY, Oriental $\ldots \ldots \ldots \ldots \ldots \ldots$

PRIMULA, Obconica Gigantea, Apple Blossom, Kermesina, Rosea, Alba or Coerulea, 1000 seeds 50c.. Mixed colors . ...............

SALPIGLOSIS, Emperor ......... SCABIOSA, Caucasica, Lavender or White $\ldots \ldots \ldots \ldots \ldots \ldots \ldots \ldots$ STOKESIA, Cyanea, Blue or White SHASTA DAISY, Alaska, California or Westralia, $1 / 4$ oz., $50 \mathrm{c} . \ldots$. SWEET WILLIAM, Pink Beauty.. SWEET WILLIAM, Mixed ........ ZINNIA, Robusta or Giant; Choice Mixed. Per pound. $\$ 7.50 \ldots \ldots$. 